\title{
RETRACTED ARTICLE: Numerical investigation to observe combined effect of propeller boss cap fin (PBCF) and propeller duct to improve propeller efficiency
}

\author{
Pritam Majumder ${ }^{1}$
}

Received: 22 March 2020 / Accepted: 21 September 2020 / Published online: 7 October 2020

(C) The Japan Society of Naval Architects and Ocean Engineers (JASNAOE) 2020

The Editor-in-Chief has retracted this article [1] on account of errors found in the manuscript.

It was found that numerical simulations have been performed using the propeller configuration that is adopted for open water test however with the velocity coming from the opposite direction. Hence all findings are inaccurate.

Pritam Majumder and Subhendu Maity disagree with this retraction. Dileep Kumar Avanapu did not respond to correspondence about this retraction.

The online version of this article contains the full text of the retracted article as Supplementary Information.
Supplementary Information The online version of this article https:// doi.org/10.1007/s00773-020-00775-w contains supplementary material, which is available to authorized users.

\section{Reference}

1. Majumder P, Avanapu DK, Maity S (2020) Numerical investigation to observe combined effect of propeller boss cap fin (PBCF) and propeller duct to improve propeller efficiency. J Mar Sci Technol. https://doi.org/10.1007/s00773-020-00775-w

Publisher's Note Springer Nature remains neutral with regard to jurisdictional claims in published maps and institutional affiliations.
Pritam Majumder

majumderpritam08@gmail.com

1 Department of Mechanical Engineering, NIT Meghalaya, Shillong 793003, India 